\title{
DARK MATTER SUBSTRUCTURE DETECTION USING SPATIALLY RESOLVED SPECTROSCOPY OF LENSED DUSTY GALAXIES
}

\author{
Yashar Hezaveh $^{1}$, Neal Dalal $^{2}$, Gilbert Holder $^{1}$, Michael Kuhlen $^{3}$, \\ Daniel Marrone ${ }^{4}$, Norman Murray ${ }^{5,7}$, AND Joaquin Vieira ${ }^{6}$ \\ ${ }^{1}$ Department of Physics, McGill University, 3600 Rue University, Montreal, Quebec H3A 2T8, Canada \\ ${ }^{2}$ Astronomy Department, University of Illinois at Urbana-Champaign, 1002 West Green Street, Urbana, IL 61801, USA \\ ${ }^{3}$ Theoretical Astrophysics Center, University of California, Berkeley, CA 94720, USA \\ ${ }^{4}$ Steward Observatory, University of Arizona, 933 North Cherry Avenue, Tucson, AZ 85721, USA \\ ${ }^{5}$ CITA, University of Toronto, 60 St. George Street, Toronto, ON M5S 3H8, Canada \\ ${ }^{6}$ California Institute of Technology, 1200 East California Blvd, MC 249-17, Pasadena, CA 91125, USA \\ Received 2012 October 15; accepted 2013 February 15; published 2013 March 19
}

\begin{abstract}
We investigate how strong lensing of dusty, star-forming galaxies (DSFGs) by foreground galaxies can be used as a probe of dark matter halo substructure. We find that spatially resolved spectroscopy of lensed sources allows dramatic improvements to measurements of lens parameters. In particular, we find that modeling of the full, threedimensional (angular position and radial velocity) data can significantly facilitate substructure detection, increasing the sensitivity of observables to lower mass subhalos. We carry out simulations of lensed dusty sources observed by early ALMA (Cycle 1) and use a Fisher matrix analysis to study the parameter degeneracies and mass detection limits of this method. We find that even with conservative assumptions, it is possible to detect galactic dark matter subhalos of $\sim 10^{8} M_{\odot}$ with high significance in most lensed DSFGs. Specifically, we find that in typical DSFG lenses, there is a $\sim 55 \%$ probability of detecting a substructure with $M>10^{8} M_{\odot}$ with more than $5 \sigma$ detection significance in each lens, if the abundance of substructure is consistent with previous lensing results. The full ALMA array, with its significantly enhanced sensitivity and resolution, should improve these estimates considerably. Given the sample of $\sim 100$ lenses provided by surveys such as the South Pole Telescope, our understanding of dark matter substructure in typical galaxy halos is poised to improve dramatically over the next few years.
\end{abstract}

Key words: dark matter - galaxies: dwarf - galaxies: luminosity function, mass function - galaxies: structure gravitational lensing: strong

Online-only material: color figures

\section{INTRODUCTION}

The inflationary $\Lambda \mathrm{CDM}$ model has proven spectacularly successful in describing the observed large-scale structure of the universe. Precise measurements of the mean expansion history of the universe (e.g., Suzuki et al. 2012), the anisotropies of the cosmic microwave background (Komatsu et al. 2011), and the clustering of galaxies at low redshift $z \lesssim 1$ (e.g., Reid et al. 2012; Sanchez et al. 2012) are all consistent with a simple scenario involving a nearly scale-invariant spectrum of curvature perturbations generated during inflation, whose growth over cosmic time is governed by gravitational instability.

While measurements of large-scale $(\gtrsim 10 \mathrm{Mpc})$ structure have yielded the most stringent constraints on this cosmological model, there is considerable information to be gleaned from observations of structure on smaller scales as well. For example, the detailed shape of the inflationary potential influences the shape of the primordial power spectrum (e.g., Dodelson 2003), motivating efforts to measure the scalar spectral index $n_{s}$ and its running $d n_{s} / d \log k$. For example, a sharp feature in the inflationary potential will generate features in the primordial power spectrum, breaking its near scale invariance (Kamionkowski \& Liddle 2000). In addition, the particle physics of dark matter affects the shape of the matter transfer functions, leading to a Jeans-like suppression of structure below the free-streaming scale of the dark matter particles. In weakly interacting massive particle like scenarios, this free-streaming scale is as small as

\footnotetext{
7 Canada Research Chair in Astrophysics.
}

one comoving parsec (Loeb \& Zaldarriaga 2005); however, in alternative dark matter models, this damping scale can be much larger (e.g., Bode et al. 2001; Abazajian 2006; Cembranos et al. 2005; Kaplinghat 2005; Cyr-Racine \& Sigurdson 2012). Accordingly, measurements of the small-scale power spectrum can in principle provide a wealth of information about the physics of the early universe.

To date, the most constraining bounds on the small-scale power spectrum have been derived from observations of the Ly $\alpha$ forest (Seljak et al. 2006). However, it will be difficult for future LyAF observations to improve significantly on existing bounds because current, high-resolution spectra already have resolution approaching the Jeans scale of the intergalactic medium at $z \sim$ $2-3$. Another probe of small-scale structure is the abundance of low-mass dark matter halos and subhalos. High-resolution $\mathrm{N}$-body simulations (Diemand et al. 2007, 2008; Stadel et al. 2009; Navarro et al. 2010) have revealed that dark matter halos in hierarchical cold dark matter (CDM) cosmologies are expected to contain copious substructure, primarily in the form of gravitationally self-bound, dynamically cold subhalos with a spectrum of masses. The abundance of these subhalos is expected to depend on the amplitude and shape of the smallscale power spectrum (e.g., Zentner \& Bullock 2003), meaning that measurements of halo substructure should constrain earlyuniverse physics.

Numerous groups have attempted to constrain the abundance of low-mass subhalos by detecting faint, dark-matter-dominated dwarf satellite galaxies in the Local Group (see Kravtsov 2010, for a review). Despite heroic efforts, the number of detected 
dwarf satellites falls well below the expected abundance of lowmass subhalos for a typical galactic halo. This discrepancy has been termed the "Satellite Problem" for CDM models, and has led to considerable speculation that the physics of dark matter might not be described well by the idealized, non-interacting, absolutely cold CDM model. However, astrophysical processes can plausibly suppress the star formation efficiency of lowmass halos and subhalos, rendering many of them invisible to optical surveys. To distinguish between astrophysical solutions and particle-physics solutions to the Satellite Problem, a purely gravitational method for detecting potentially dark subhalos is required. One possible means of detecting dark substructure in galactic halos is to search for its dynamical effects on cold stellar streams (Carlberg et al. 2011; Yoon et al. 2011). Another gravitational probe of dark matter substructure is strong gravitational lensing, which is the focus of this work.

Mao \& Schneider (1998) were the first to suggest that strong gravitational lenses could be used to detect dark matter substructure through anomalous flux ratios of multiply imaged quasars. Many groups subsequently followed up on this idea, both theoretically and observationally (Dalal \& Kochanek 2002; Kochanek \& Dalal 2004; Chiba et al. 2005; Minezaki et al. 2009; MacLeod et al. 2009; Miranda \& Macciò 2007; Chen et al. 2007, 2011; Rozo et al. 2007; Keeton et al. 2006; Chen et al. 2003; Moustakas \& Metcalf 2003; Metcalf et al. 2004; Metcalf 2002; Schechter \& Wambsganss 2002; Koopmans et al. 2002; Metcalf \& Madau 2001; Keeton et al. 2003; More et al. 2009; Fadely \& Keeton 2011; Keeton et al. 2005; Xu et al. 2009; Vegetti et al. 2012). In particular, Dalal \& Kochanek (2002) analyzed a sample of quadruply imaged radio quasars from the CLASS survey (Browne et al. 2003), and from the preponderance of flux anomalies in these lenses, concluded that a large fraction $(\sim 1 \%-2 \%)$ of the projected mass at the Einstein radius, $\sim 5 \mathrm{kpc}$, must be in the form of local substructure. The uncertainties on this measurement were quite large, however, due to the small sample size used in that analysis. Subsequent work has not significantly improved the bounds on substructure from strong lensing, mainly due to the difficulties in using optical lenses to study substructure (Kochanek \& Dalal 2004); however, Vegetti et al. (2012) report a recent subhalo detection using an extended optical galaxy-galaxy lens system.

Fortunately, in recent years, a new class of lensed sources has been detected: dusty, star-forming galaxies (DSFGs) at high redshift, $z \sim 2-5$ (Vieira et al. 2010; Negrello et al. 2010). DSFGs are a class of luminous and prodigiously star-forming galaxies located at high redshift $(z>1)$. They are enshrouded in dust (Blain et al. 2002; Lagache et al. 2005) and contain massive reservoirs of molecular gas (Greve et al. 2005; Carilli et al. 2011). The molecular gas in these galaxies is excited by the intense high energy emission of the active star-forming regions. Most commonly, $\mathrm{CO}, \mathrm{H}_{2} \mathrm{O}, \mathrm{HCN}$, and $\mathrm{HCO}+$ are the molecular lines observed in these galaxies (Solomon \& Vanden Bout 2005). In addition to molecular lines, atomic fine structure lines such as ionized carbon ([C II]) have long been known to be a dominant cooling mechanism for DSFGs, and in some cases can account for $10^{-3}$ of their total infrared luminosity (Stacey et al. 1991). The intrinsic sizes and morphologies of DSFGs are not well understood, but some studies (Chapman et al. 2004; Tacconi et al. 2006; Younger et al. 2008; Engel et al. 2010) suggest typical radii of $\sim 1 \mathrm{kpc}$ for these galaxies. The submm emission from DSFGs is believed to be dominated by multiple compact clumps of intense star formation spread out over the extent of the galaxy. For example, Swinbank et al. (2010) derive upper limits of $\sim 200 \mathrm{pc}$ for the diameters (FWHM) of star-forming clumps in a lensed DSFG.

The brightest DSFGs were predicted to be strongly lensed (Blain 1996; Negrello et al. 2007; Hezaveh \& Holder 2011). This prediction was confirmed by high-resolution follow-up imaging of the brightest extragalactic sources found by the South Pole Telescope (SPT; Carlstrom et al. 2011; Vieira et al. 2012; Hezaveh et al. 2012a) and the Herschel Space Observatory (Negrello et al. 2010). Recently, Hezaveh et al. (2012a) presented lens models for four lensed DSFGs observed with ALMA in Cycle 0. Even with very short total observing times $(\sim 20 \mathrm{~s})$ and only $\sim 15$ antennas, they were able to derive tight constraints on the mass distributions of the foreground lenses, thanks to the extreme brightness of the sources combined with ALMA's high sensitivity. The stringent constraints that are possible using snapshot observations with the small subset of telescopes available in Cycle 0 strongly suggest that the full ALMA array could provide an unprecedented view of the detailed mass distributions of lensing galaxies. In particular, ALMA observations of lensed DSFGs could revolutionize the study of dark matter halo substructure in lens galaxies.

Lensed DSFGs are a particularly attractive population for substructure studies, due to their great abundance (compared to quasar lenses). In addition, since almost all of the UV and optical emission in the DSFGs is absorbed and reradiated by dust at longer wavelengths, these sources are almost completely invisible in optical images. If substructure is detected gravitationally, then deep optical imaging would place stronger limits on the mass to light ratios of the galactic satellites than would be possible if the source galaxies were bright in optical bands. Moreover, the high redshifts of the sources permit a wide range of possible lens redshifts, potentially allowing constraints on any redshift evolution in the substructure population.

In this work, we study the feasibility of using ALMA to detect dark matter substructure in the halos of lens galaxies. In particular, we study how the spatially resolved spectroscopy provided by ALMA allows us to resolve source structures and dramatically increase the substructure detection sensitivity. In Section 2, we discuss the benefits of spectroscopically resolved interferometric observations of lensed DSFGs. In Section 3, we describe our simulations of ALMA observation of lensed DSFGs. In Sections 4 and 5, we present results of our simulations, and conclude in Section 6.

\section{SPATIALLY RESOLVED SPECTROSCOPY OF LENSED SOURCES}

One of the most important properties of lensed systems which determines the sensitivity of observations to substructure lensing is the size of the source. A source that is completely uniform across some scale $r_{\text {src }}$ will be insensitive to lensing perturbations from structures on scales small compared to $r_{\text {src }}$. When studying dark matter substructure, it is therefore advantageous to use lensed sources that intrinsically have structure on angular scales of the order of milliarcseconds, comparable to the Einstein radii of the subhalos of interest. For example, radio quasars are excellent sources for studying substructure, since their intrinsic sizes $(\theta \lesssim 0.1$ mas) are much larger than the Einstein radii of individual stars but smaller than the Einstein radii of subhalos. In contrast, optical QSO's have much smaller angular sizes, meaning that their lensed images can be affected by stellar microlensing, which can be difficult to disentangle from substructure lensing (Kochanek \& Dalal 2004; Morgan et al. 2012; Sluse et al. 2012). Similarly, typical optically bright 
galaxies have much larger angular sizes, of the order of $\sim 1^{\prime \prime}$, rendering most galaxies insensitive to substructure perturbations (however, see Vegetti \& Koopmans 2009 and Vegetti et al. 2012 for a method that uses the compact details of the structure in the source for substructure detection).

Naively, DSFGs would appear to be ill-suited as sources for substructure lensing. Typical DSFGs are believed to have physical radii of the order of $1 \mathrm{kpc}$, corresponding to an angular scale of the order of 0.2 , much larger than the Einstein radii of all but the most massive subhalos. However, these galaxies do not have a smooth and uniform morphology. They typically are believed to be composed of multiple compact knots of star formation, based both on theoretical and observational grounds (Swinbank et al. 2010). In principle, these compact clumps should be sensitive to lensing perturbations on smaller scales than the galaxy as a whole. In practice, however, the superposition of a large number of blended, overlapping source clumps becomes indistinguishable from an extended source, thereby reducing sensitivity to substructure lensing. In order to fully exploit the potential of lensed DSFGs as a probe of dark matter substructure, we require some method to decompose the source emission into its constituent compact clumps.

Fortunately, it is possible to perform such a decomposition in velocity space. The idea is analogous to the method of Moustakas \& Metcalf (2003) and Metcalf et al. (2004). Spatially resolved spectroscopic observations of DSFGs show strong velocity gradients, some indicating fast-rotating disks and others indicating major mergers (Engel et al. 2010; Riechers et al. 2011; Hodge et al. 2012). If the line-of-sight velocity offset between star-forming clumps is larger than the velocity dispersion of single clumps, then observations at different frequencies will allow us to distinguish the emission from distinct clumps. In other words, the emission in a narrow frequency window will come from a region in the source much smaller than the overall extent of the DSFG. Effectively, we can use spectroscopic resolution to enhance our spatial resolution.

Radio interferometers (such as ALMA) provide spatial and spectral resolution on interesting scales simultaneously, making these instruments ideal observatories for probing substructure. We expect a significantly enhanced sensitivity to substructure perturbations when we simultaneously model the visibilities observed in all the channels, compared to modeling the summed, channel-integrated visibility set. In the next section, we demonstrate this enhanced sensitivity using simulations of ALMA observations of lensed DSFGs.

\section{SIMULATIONS}

We simulate observations of lensed DSFGs with ALMA Cycle 1 (32 antennas) with the array in its most extended configuration, with a maximum baseline of $1.1 \mathrm{~km}$. This allows a resolution of 0 '. 16 at $350 \mathrm{GHz}$. The noise levels are calculated using the online ALMA Sensitivity Calculator for best observing conditions (1st Octile: $\mathrm{PWV}=0.5 \mathrm{~mm}, T_{\text {sys }}=90 \mathrm{~K}$ ). Note that the full ALMA array will have roughly 4 times greater sensitivity, and more than 10 times higher spatial resolution than what we have assumed in the calculations presented here. We place the source at decl. $-50: 30$ and simulate a $1 \mathrm{hr}$ observation with $10 \mathrm{~s}$ integration time. The $u v$-coverage was predicted using the "simdata" task of the Common Astronomy Software Application package (Petry et al. 2012).

The source is modeled as a collection of giant star-forming clumps. Since we focus on high-excitation lines that are found only in high-density clumps, we will neglect any diffuse emission originating from the interclump interstellar medium. In local starburst galaxies, and in high-redshift DSFGs, CO line brightness peaks at $J=6$, and at higher $J$ in active galactic nucleus dominated sources (Weiß et al. 2007; Lestrade et al. 2010; Rangwala et al. 2011). Since transitions such as CO 6-5 produce some of the brightest lines and trace the dense cores of star-forming clumps with compact morphologies, they may be the ideal molecular lines for substructure lensing detections.

The clumps are placed randomly in both spatial location and velocity, with positions drawn from a Gaussian profile with an rms of $1 \mathrm{kpc}$ and velocities drawn from a uniform distribution of width $300 \mathrm{~km} \mathrm{~s}^{-1}$. Each clump has a circular Gaussian surface brightness profile with FWHM of $d_{c}=250 \mathrm{pc}$ and a Gaussian profile in redshift space characterized by a velocity dispersion of $\sigma_{v}=30 \mathrm{~km} \mathrm{~s}^{-1}$. For simplicity, we have used random velocities for the clumps, as opposed to a rotating disk, for example. As long as the clumps are reasonably well separated in velocity space $\left(\Delta v \gtrsim \sigma_{v}\right)$, we do not expect any qualitative differences between ordered motion and disordered motion. Each source clump is therefore described by six parameters: $x$ and $y$ centroids, central velocity, radius, flux, and velocity dispersion. The source emission, summing over all clumps, is represented as a threedimensional data cube. The total intrinsic velocity-integrated flux of the entire DSFG is set to $1 \mathrm{Jy} \mathrm{km} \mathrm{s}^{-1}$ (see Bothwell et al. 2012 for similar values for the velocity-integrated CO flux of unlensed DSFGs) and distributed equally among 10 starforming clumps.

Given our source realizations, we then ray-trace to compute a new three-dimensional data cube containing the lensed images observed in each channel. We then Fourier transform each layer of the cube and predict the visibilities by interpolating the Fourier maps.

In each simulation, the main lens galaxy is modeled as a singular isothermal ellipsoid (SIE), which has a three-dimensional mass density proportional to $r^{-2}$, and projected surface density

$$
\Sigma(x, y)=\frac{\sqrt{q} v^{2}}{2 G}\left(\frac{x^{2}+q^{2} y^{2}}{r_{\mathrm{E}}^{2}}\right)^{-1 / 2},
$$

where $x$ and $y$ are coordinates oriented along the principal axes of the surface density measured relative to the lens centroid, $v$ is the velocity dispersion along the line of sight, $q$ is the axis ratio, and $r_{\mathrm{E}}$ is the Einstein radius of the lens,

$$
r_{\mathrm{E}}=4 \pi \frac{v^{2}}{c^{2}} \frac{D_{d} D_{d s}}{D_{s}}
$$

(Kormann et al. 1994). The SIE therefore is described by five parameters: the centroid $x_{c}, y_{c}$, velocity dispersion $v$, axis ratio $q$, and orientation $\theta_{q}$. We simulate lenses with Einstein radius mass $M_{\mathrm{E}}=\pi \Sigma_{c r} r_{\mathrm{E}}^{2}$ of $4 \times 10^{11} M_{\odot}$ placed at $z_{d}=0.5$ and place the source at $z=2$. In addition to this main lens, we allow for external shear, described by an amplitude $|\gamma|$ and orientation $\theta_{\gamma}$. The smooth mass model therefore has seven parameters describing the lens. We follow previous work and model dark matter subhalos using the pseudo-Jaffe density profile (Muñoz et al. 2001),

$$
\kappa(x)=\frac{1}{2 x}-\frac{1}{2 \sqrt{x^{2}+x_{t}^{2}}},
$$

where $x=r / r_{\mathrm{E}}$ and $x_{t}=r_{t} / r_{\mathrm{E}}$ are the radius and the tidal truncation radius, respectively, in units of the subhalo's Einstein radius, and $\kappa=\Sigma(r) / \Sigma_{c r}$ is the dimensionless surface 

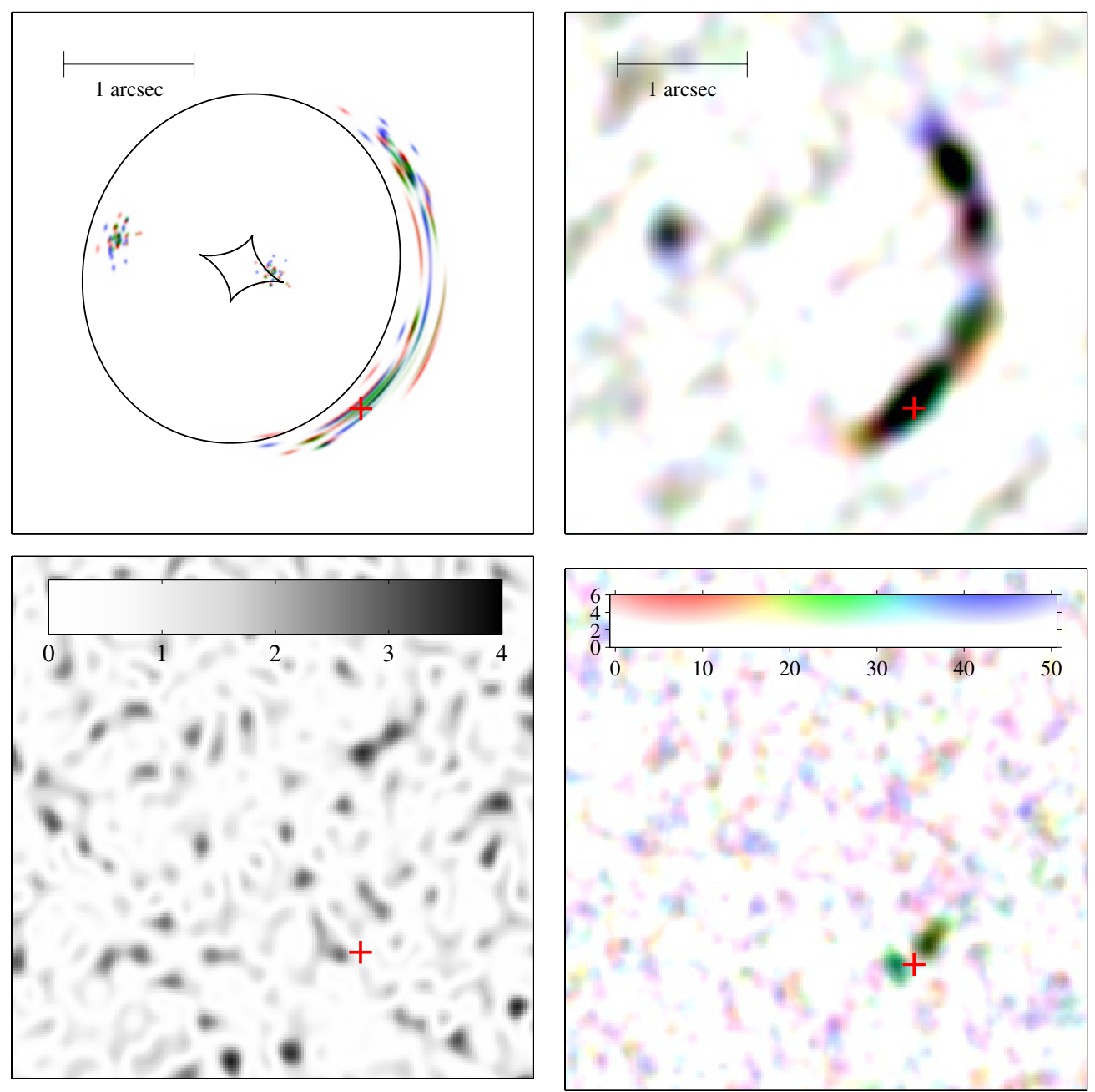

Figure 1. Top left: the source model and the lensed images of a clumpy source. The black curves show the tangential and radial caustics for the unperturbed macro model, and the red + symbol shows the location of an additional subhalo of mass $M=10^{8} M_{\odot}$. Top right: the dirty image observed by ALMA. Bottom left: residuals of channel-integrated dirty images between a smooth model and noisy perturbed observation. The gray scale is in units of noise rms. Bottom right: color residuals of the dirty images. The 50 observed channels are mapped to RGB colors as illustrated by the color bar. The $y$-axis of the color bar shows the intensity in units of image noise rms in each channel.

(A color version of this figure is available in the online journal.)

density expressed in units of the lensing critical surface density (Schneider et al. 1992).

Figures 1 and 2 illustrate the increased sensitivity to substructure provided by spectroscopically resolved data. Figure 1 shows an example of ALMA observations of a lensed DSFG, both for spectroscopically resolved visibilities and channelintegrated visibilities. The gray scale and colored panels show the residuals from the best-fitting smooth lens models for each case, and the substructure perturbation clearly stands out more readily in velocity space. In Figure 2, we show the substructure parameter errors derived from simulated observations of another lensed DSFG, whose properties were chosen to be representative of the lenses found in Hezaveh et al. (2012a). The parameter uncertainties plotted in this figure were estimated by a Fisher matrix calculation; we marginalize over a considerably larger number of nuisance parameters describing the source emission when fitting spectroscopically resolved visibilities, compared to fitting the channel-integrated visibilities. Despite the increased number of marginalized nuisance parameters, the parameter uncertainties are considerably reduced when we utilize the full, velocity-resolved data cube (compare gray versus blue contours). This is true for the "macro model" param- eters describing the smooth lens, and for the subhalo parameters as well.

ALMA observations will therefore clearly improve our ability to characterize substructure in strong lenses. As Figure 2 demonstrates, the properties of an $M=10^{8} M_{\odot}$ subhalo can be determined with high precision, especially when velocity information is utilized in the model fits. However, before undertaking a systematic study of subhalo parameter measurements, it is important to estimate the substructure detection rates expected in ALMA observations of typical lens systems. Will detections like the subhalo shown in Figure 2 (a $6.8 \sigma$ detection) be common or rare in DSFG lenses? The focus of this paper is to demonstrate the benefits of velocity fitting and to quantify the expected substructure detection rates using velocity fitting with ALMA.

We define the significance of a subhalo detection in terms of the residual $\chi^{2}$ between the best-fit smooth mass model and the simulated observations. In our simulations, we start with a smooth macro model and add deflections from a single subhalo during the ray-trace. We then model the simulated observations using only the smooth mass model, with no substructure parameters, allowing the macro model parameters to adjust in order to account for the substructure perturbations. 


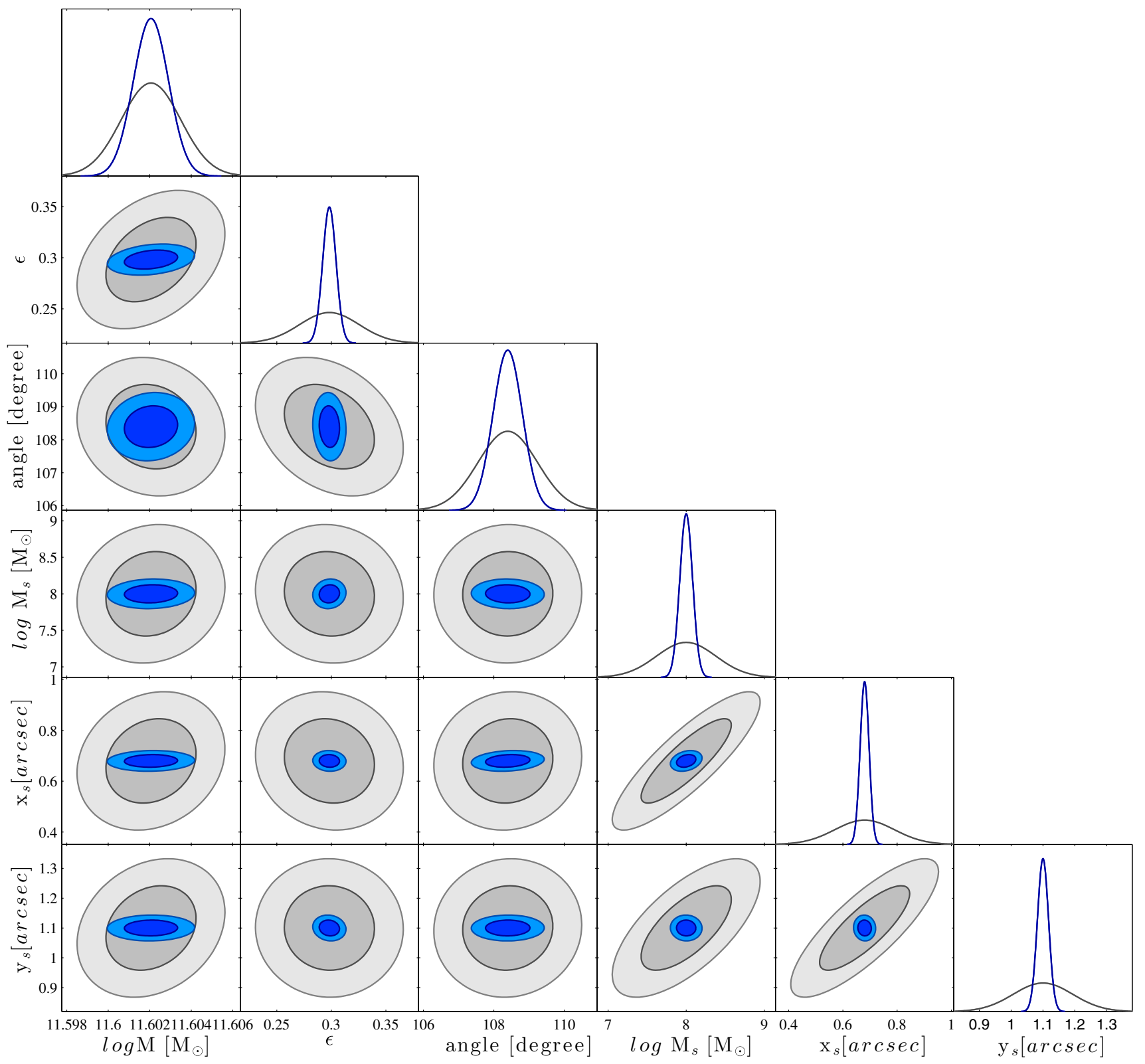

Figure 2. Fisher matrix error forecasts. We simulate ALMA observations of a lensed DSFG, including substructure perturbations from a single subhalo of mass $M=10^{8} M_{\odot}$ in the lens mass distribution, and compute the Fisher matrix for all the lens (including substructure) and source parameters. We have marginalized over all parameters that are not shown in the figure, including 5 parameters for each of the 10 sources (see the text), external shear, and the centroid of the main lens. The parameters plotted here are $M$ (Einstein mass of the main lens), $\epsilon$ (ellipticity of the main lens), angle (orientation angle of the main lens), $M_{s}$ (total mass of the subhalo), $x_{s}$, and $y_{s}$ (the position of the subhalo). Gray contours correspond to fitting the channel-integrated flux, and blue contours correspond to fitting all channels simultaneously.

(A color version of this figure is available in the online journal.)

We follow Dalal \& Kochanek (2002) and use linear perturbation theory to determine the parameters of the best-fitting smooth macro model. We characterize the smooth macro model with a parameter set $\boldsymbol{p}$ (including the source profile parameters). For some fiducial set of parameters $\boldsymbol{p}_{0}$, we obtain observables $\boldsymbol{O}$ (a vector of real and imaginary visibilities) and write the penalty function

$$
\chi^{2}=\delta O_{i}\left(\mathbf{C}^{-1}\right)_{i j} \delta O_{j},
$$

where summation over repeated indices is understood and $\mathbf{C}$ is the noise covariance matrix. If our observables are the visibilities, then $\mathbf{C}$ is diagonal.

A perturbation to the model parameters $\delta \boldsymbol{p}$ generates perturbations to the observables given by $\delta O_{i}=\left(\partial O_{i} / \partial p_{j}\right) \delta p_{j}$. Given a current data residual $\delta \boldsymbol{O}$, the addition of these parameter adjustments changes the $\chi^{2}$ to

$$
\chi^{2}=\left[\delta O_{i}+\delta p_{k} \frac{\partial O_{i}}{\partial p_{k}}\right]\left(\mathbf{C}^{-1}\right)_{i j}\left[\delta O_{j}+\frac{\partial O_{j}}{\partial p_{l}} \delta p_{l}\right] .
$$

To find the parameter set $\boldsymbol{p}$ that minimizes this function, we set $\partial \chi^{2} / \partial p_{i}=0$ and solve for $\delta p_{i}$

$$
\delta p_{l}=-\left(\mathbf{F}^{-1}\right)_{l k} \frac{\partial O_{j}}{\partial p_{k}}\left(\mathbf{C}^{-1}\right)_{j i} \delta O_{i},
$$

where

$$
F_{i j}=\frac{\partial O_{k}}{\partial p_{i}}\left(\mathbf{C}^{-1}\right)_{k l} \frac{\partial O_{l}}{\partial p_{j}}
$$

is the Fisher matrix for the smooth model. 

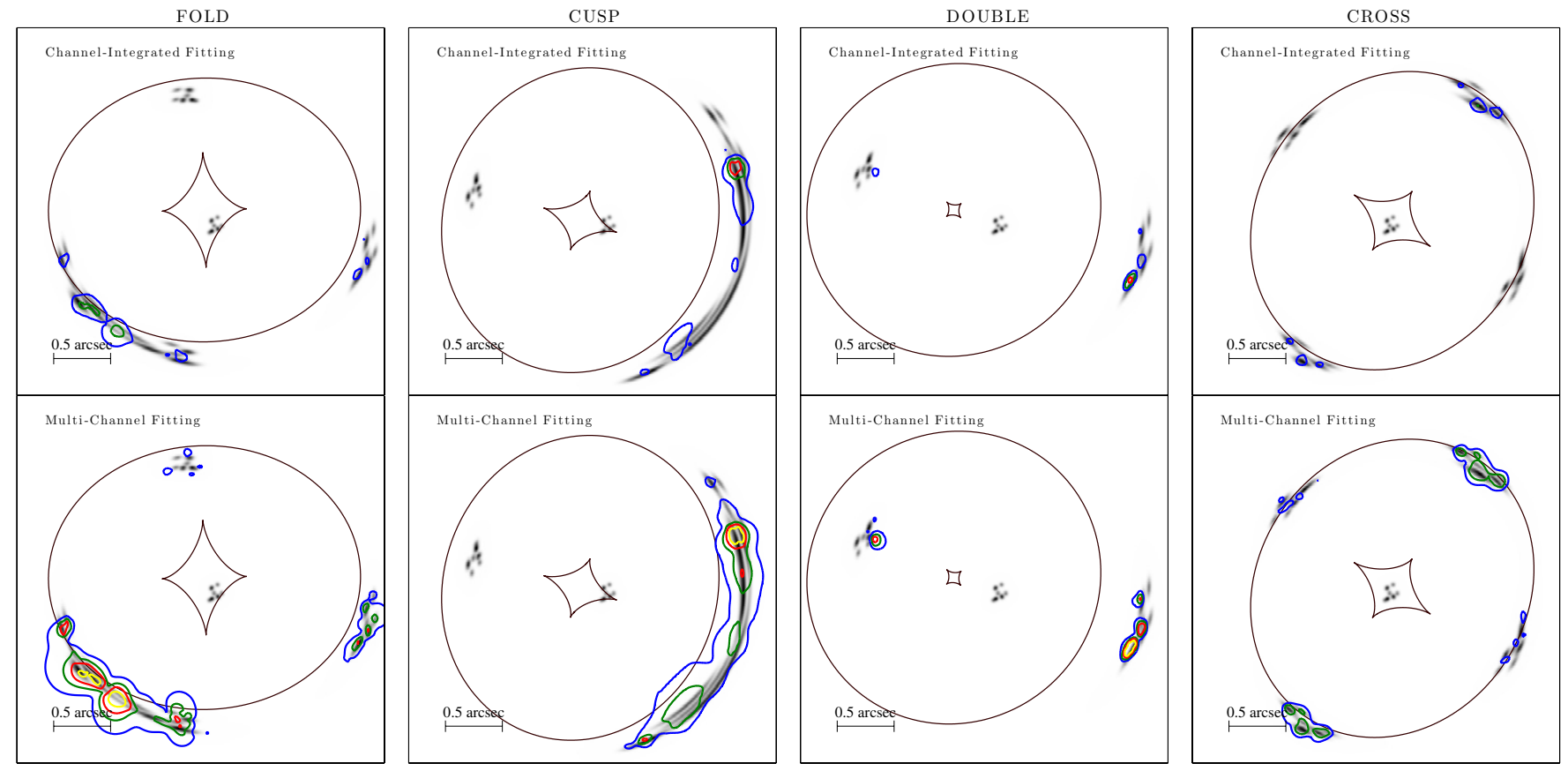

Figure 3. Substructure detection cross-sections. Contours show the detection significance for substructure as a function of position for a subhalo of mass $M=10^{8} M_{\odot}$ The different colors correspond to 3 (blue), 5 (green), 7 (red), and 10 (yellow) sigma detections. Columns correspond to different lensing configurations labeled on top (fold, cusp double, and cross from left to right). The gray-scale image shows the model lensed images. The unlensed source is also plotted at the center. The black curves show the tangential and radial caustics, not the critical curves. The bottom row shows the detection significance when fitting to all channels simultaneously, while the top row shows detection significance for channel-integrated visibilities.

(A color version of this figure is available in the online journal.)

Using this procedure, we choose a smooth macro model and calculate the matrix $\partial O / \partial p$ by finite differencing. This matrix has dimensions $n_{\mathrm{obs}} \times n_{\mathrm{par}}$, where $n_{\mathrm{obs}}=18$ million is the number of observables (real and imaginary visibilities in 50 channels for a $1 \mathrm{hr}$ observation) and $n_{\mathrm{par}}=57$ is the number of source parameters ( 7 for the lens model and 5 parameters $\mathrm{each}^{8}$ for 10 independent source clumps). We use this matrix to calculate the Fisher matrix F using Equation (7). We add a single subhalo to the current smooth model, predict the new visibilities, and use Equation (6) to find the parameter adjustments to the smooth model that minimizes $\chi^{2}$. We then simulate a smooth macro lens observation with parameters $\boldsymbol{p}+\delta \boldsymbol{p}$ and use the new $\chi^{2}$ between this best-fit model and the original simulated observation as the likelihood of a detection.

We simulate four different lensing configurations labeled "fold," "cusp," "double," and "cross." All configurations have parameters that are typical for strong lensing systems. As before, the background source is chosen to consist of 10 Gaussian clumps, a diameter (FWHM) of $250 \mathrm{pc}$, and a velocity dispersion of $30 \mathrm{~km} \mathrm{~s}^{-1}$. These values are chosen to be consistent with well-resolved observations of molecular gas clumps in lensed DSFGs (Swinbank et al. 2010, 2011). Our sensitivity to substructure depends strongly on the sizes of these clumps, as discussed in Section 5. For each configuration, we define a Cartesian grid of substructure positions. We place a single subhalo at each location and use the above procedure to measure the detection significance of substructure with mass $M$ as a function of position. The area inside a contour of specified $\chi^{2}$ then defines the detection cross-section for each significance level.

\footnotetext{
8 We did not vary each clump's velocity dispersion $\sigma$, since we did not expect it to be degenerate with any parameters of the lens mass model.
}

We perform simulations for 20 different substructure masses, between $10^{7}-10^{9} M_{\odot}$. Our results, shown in Figures 4 and 5, are discussed in the next section.

\section{RESULTS}

We define the substructure detection cross-section as the area of the sky (e.g., in square arcsec) inside of which a subhalo can be detected with a given minimum significance. We compute this cross-section by calculating the detection significance, defined in Section 3, as a function of subhalo position. Figure 3 shows the detection cross-sections at 3,5, 7, and 10 sigma significance for an $M=10^{8} M_{\odot}$ subhalo, for four different macro lens configurations. The top row shows this cross-section when the channels are integrated before fitting and the bottom panel shows the cross-section when fitting to each of the channels individually. The different extent of the detection cross-section for different lensing configurations suggests that there is a higher probability of detecting subhalos in high-magnification fold and cusp image configurations compared to low-magnification cross or double configurations. Nonetheless, the low-magnification lenses retain some sensitivity to substructure. This is unlike the case of two-image quasar lenses, which generally lack sufficient constraints to permit substructure detection. For DSFG lenses, the presence of multiple source clumps provides enough constraints on the mass model to permit substructure detection in favorable configurations.

We perform similar simulations for other subhalo masses ranging between $10^{7} M_{\odot}$ and $10^{9} M_{\odot}$, and calculate the total cross-section areas for each mass bin. Figure 4 shows these cross-sections as a function of mass for each lensing configuration. We have only plotted the cross-sections derived from channel-fitting, having already established that the 

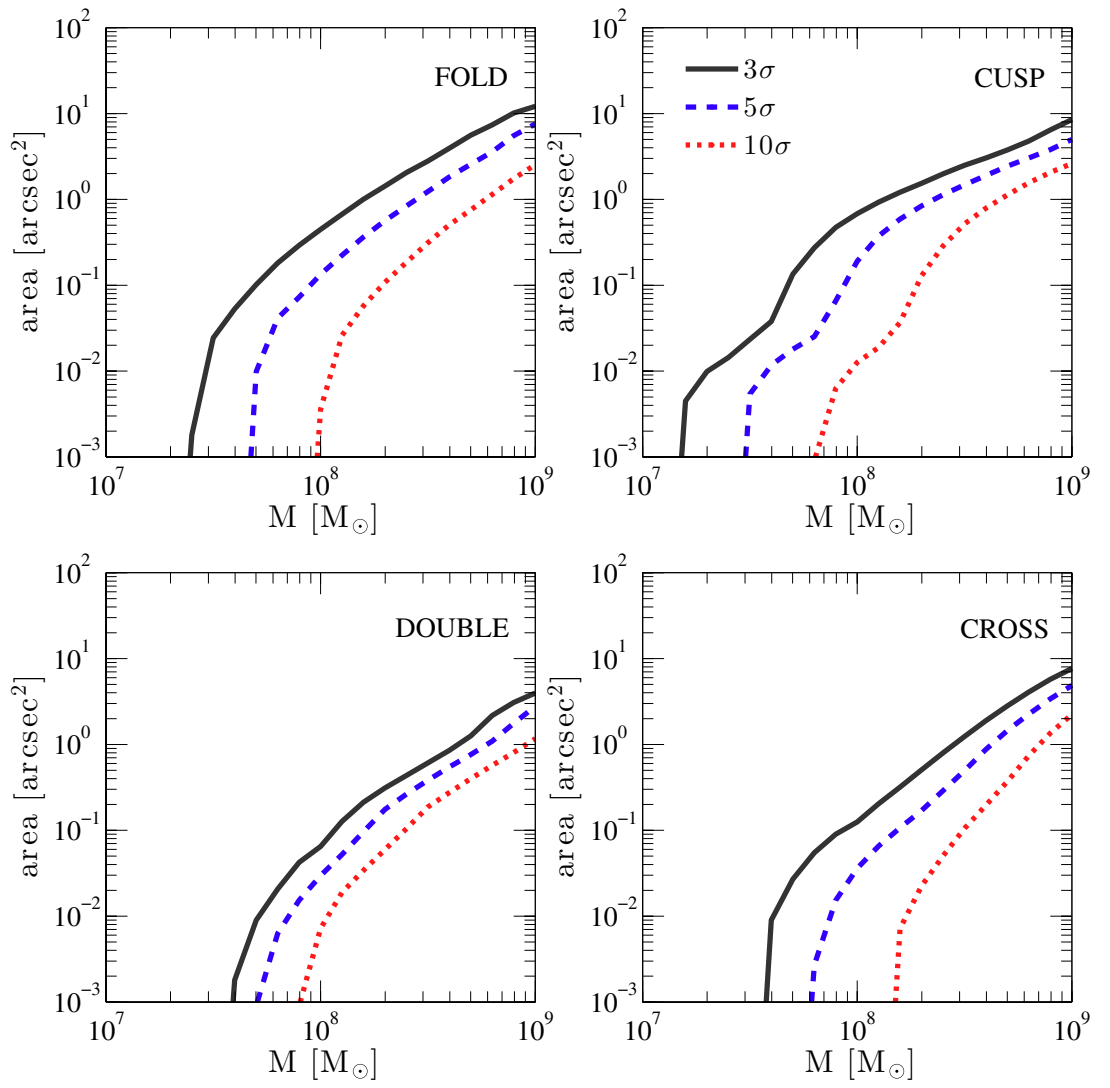

Figure 4. Detection cross-section for subhalos as a function of mass. Each panel corresponds to a different macro lens image configuration. The curves show the area of $3 \sigma$ (solid black), $5 \sigma$ (dashed blue), and $10 \sigma$ (dotted red) detection significance.

(A color version of this figure is available in the online journal.)
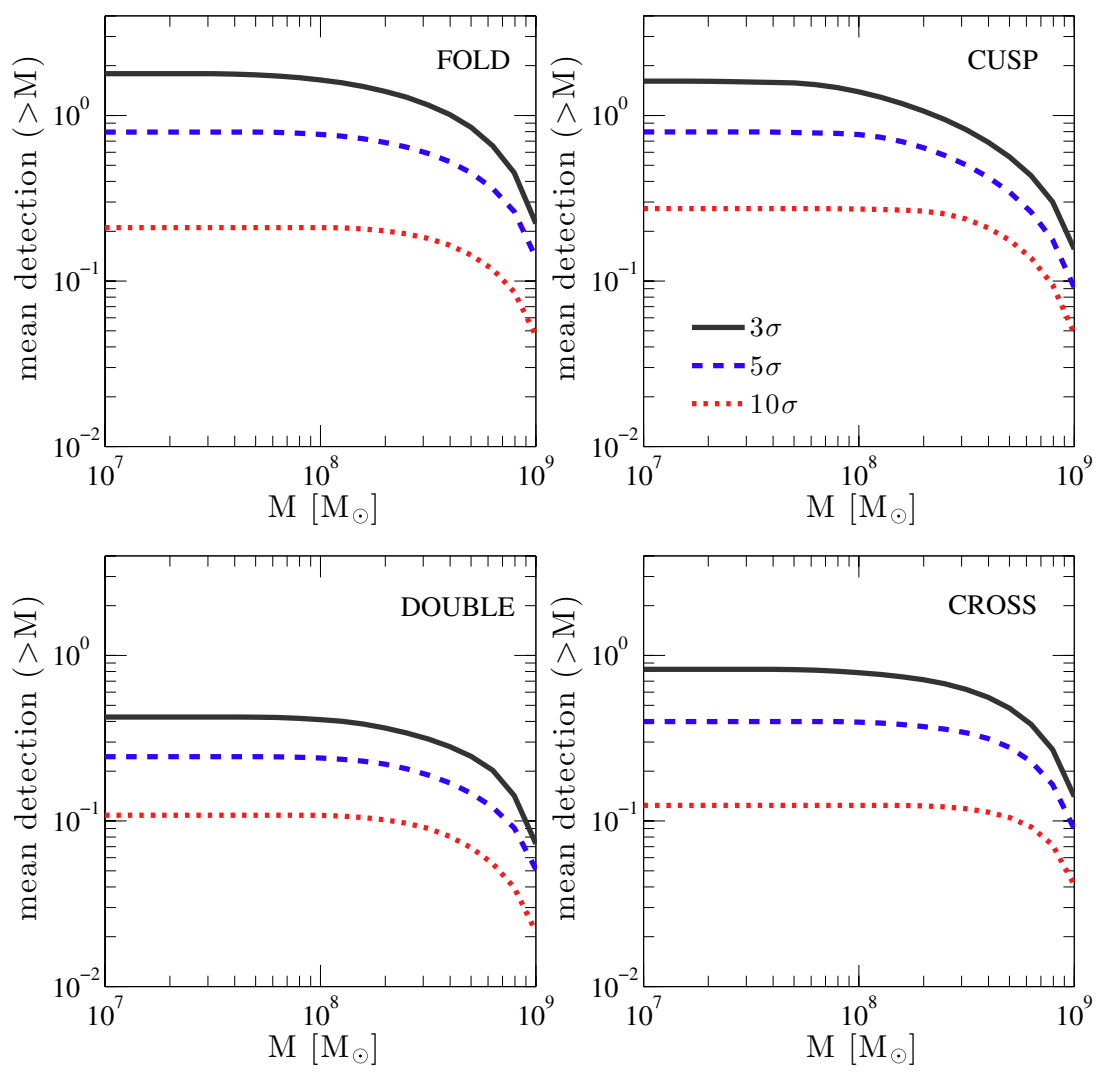

Figure 5. Left: cumulative mean number of detections of subhalos of mass greater than $M$. The curves show the number of detections with $3 \sigma$ (solid black), $5 \sigma$ (dashed blue), and $10 \sigma$ (dotted red) significance.

(A color version of this figure is available in the online journal.) 
detection sensitivity will be considerably diminished for channel-integrated data.

These cross-sections, in combination with a substructure mass function, can be used to estimate the expected number of detected subhalos for each DSFG lens. We assume a subhalo mass function with a slope of $d \log N / d \log M=-1.9$, an upper mass bound of $10^{9} M_{\odot}$, and an overall normalization placed by setting the mass fraction of substructure inside the Einstein radius of the macro lens to $f=1 \%$, consistent with results of the quasar lensing analysis of Dalal \& Kochanek (2002). Multiplying the sky number density of subhalos with masses between $M$ and $M+\delta M$ by the detection cross-section of mass $M$ gives the average number of detections with the given significance, assuming a uniform distribution of substructure over the strong lensing region. Figure 5 shows the cumulative number of detections for the four lensing configurations. Note that configurations with high magnification (the fold and cusp configurations) give more than one detected subhalo on average (at $3 \sigma$ confidence) for each lensing system. This somewhat invalidates our treatment, which explicitly assumes that only a single subhalo provides perturbations. In contrast, the collective perturbations of multiple subhalos must be simultaneously treated. In forthcoming work, we will analyze the perturbations from the population of substructure inside dark matter halos.

\section{DISCUSSION}

In the simulations presented in this work, we have assumed a fixed source morphology with reasonably conservative parameters describing the DSFGs. In this section, we briefly explore the sensitivity of our results to the assumed properties of the source clumps.

As discussed above, one of the most important parameters describing the source is its angular size. In the calculations we have presented so far, we have assumed a physical size of $250 \mathrm{pc}$ (FWHM) for the source clumps. We chose this value as a conservative upper bound, given the results of Swinbank et al. (2010). The CO-emitting regions of the star-forming clumps could be significantly smaller than this assumed size, however. Upcoming ALMA observations of DSFGs should resolve the question of the intrinsic source size. Even if the source clumps are not significantly smaller than our assumed values, observations of other molecular lines besides CO lines could reveal much smaller source sizes and different source morphologies (Carilli et al. 2011; Riechers et al. 2011; Combes et al. 2012). Transitions of $\mathrm{H}_{2} \mathrm{O}, \mathrm{HCN}$, and $\mathrm{HCO}^{+}$have higher critical densities than $\mathrm{CO}$ at similar observing frequencies. These lines trace dense gas in the active star-forming regions (Downes \& Solomon 1998) and may have fluxes as high as $25 \%$ of the CO flux (Gao \& Solomon 2004) or more (Riechers et al. 2010). Because they are confined to smaller regions than lowand possibly high- $J \mathrm{CO}$, they may increase the sensitivity to lower mass subhalos. To demonstrate this enhanced sensitivity, we have repeated the fold configuration simulations presented in Figure 4 with identical parameters, but using smaller source clumps (150 pc FWHM). The red dashed curve in the top panel of Figure 6 shows the $5 \sigma$ detection cross-sections of subhalos with masses ranging from $10^{7}$ to $10^{9} M_{\odot}$. The blue shade corresponds to the larger clump sizes presented in Figure 4. The bottom panel shows the resulting increase in the mean number of detected subhalos, demonstrating that different molecular lines originating from different morphological structures of varying sizes exhibit different levels of sensitivity to substructure.
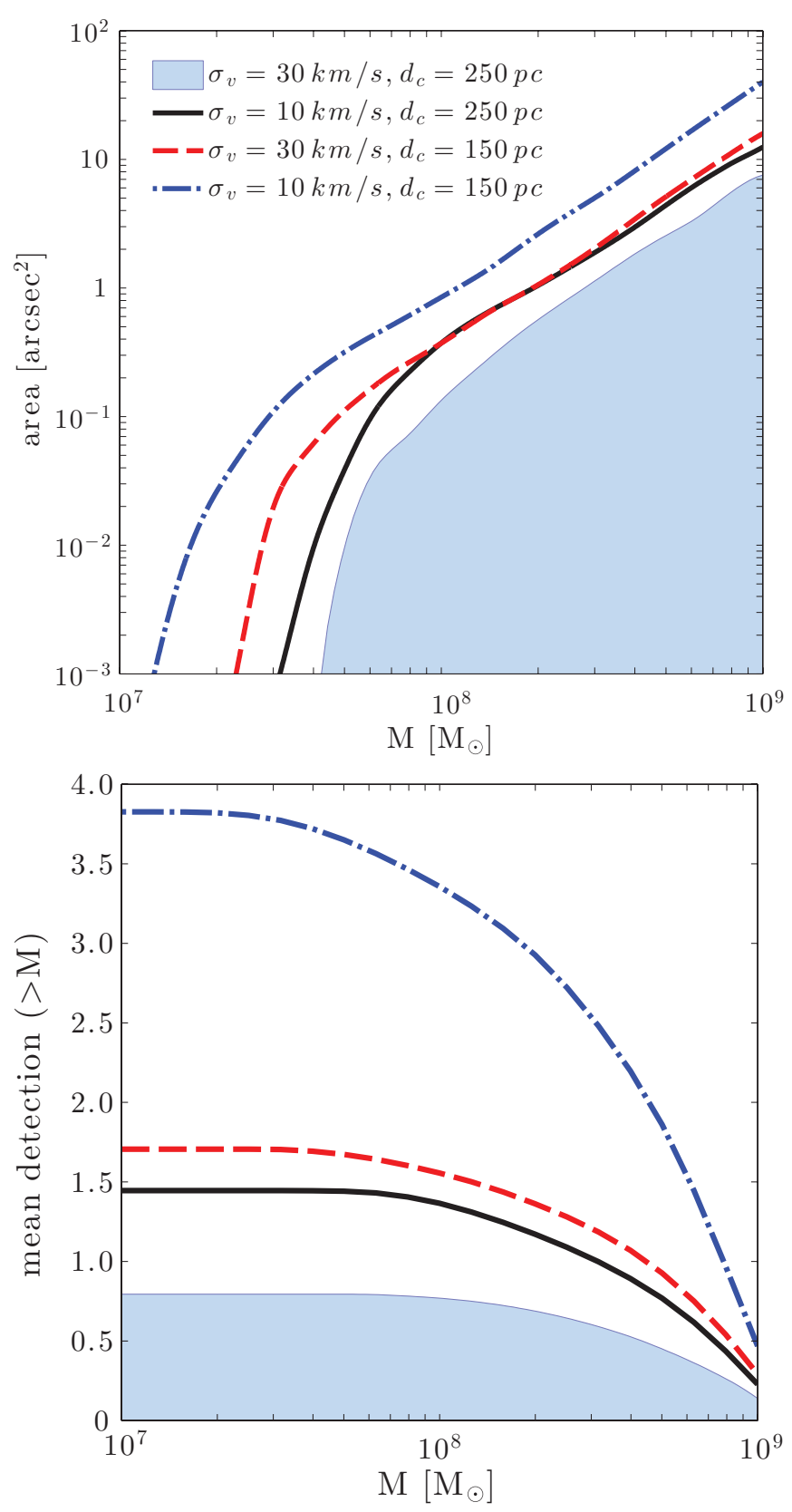

Figure 6. Detection sensitivity as a function of source properties. Here, we show the improvement in detection significance when observing DSFGs composed of smaller clumps (red dashed curves), clumps with lower velocity dispersion (solid black curves), or both (dot-dashed blue curves), compared to our fiducial source parameters (blue shaded region), for the fold configuration. The top panel shows the detection cross-section, and the bottom panel shows the mean cumulative number of detections. All curves are shown at $5 \sigma$ detection significance. Smaller clumps could correspond to observing dense-gas tracers such as $\mathrm{HCN}$.

(A color version of this figure is available in the online journal.)

Just as a smaller angular size improves the sensitivity to substructure, narrower linewidths also help resolve the emission from distinct clumps. If the intrinsic velocity dispersion of each star-forming clump is lowered, then fewer clumps appear simultaneously in each channel. This effectively shrinks the angular extent of the emitting region in each channel, increasing the substructure detection sensitivity. In Figure 6, the black solid curves show the $5 \sigma$ detection cross-section (top) and mean cumulative detection numbers (bottom) of a DSFG composed of clumps with a velocity dispersion of $10 \mathrm{~km} \mathrm{~s}^{-1}$ (Hopkins 
et al. 2012). The key factor here is the relative velocity offset of clumps with respect to each other, in units of their velocity dispersion. The blue dot-dashed curve in Figure 6 shows the detection improvements for simulations with both smaller clumps and lower velocity dispersion. As the bottom panel of Figure 6 illustrates, for a source with these parameters, we expect to detect more than one subhalo in a given lens, if the substructure abundance is consistent with previous lensing analyses.

Figure 6 demonstrates that our sensitivity to substructure depends on the clump morphology, both in real space and in velocity space. We have chosen fiducial values for these source parameters to be consistent with current measurements of the intrinsic properties of DSFGs. The unlensed DSFGs presented in Engel et al. (2010) showed either signatures of merging systems or fast rotating compact morphologies. Comparing the values of galaxy-scale velocity dispersions reported in Engel et al. (2010), which range between 200 and $1000 \mathrm{~km} \mathrm{~s}^{-1}$ (FWHM), with the $\sim 10 \mathrm{~km} \mathrm{~s}^{-1}$ dispersions expected for single clumps (Hopkins et al. 2012), indicates that all the DSFGs in their sample must contain several components with significant velocity offsets. Similarly, the double peak velocity profile presented in Hodge et al. (2012) is indicative of large velocity offsets between multiple components. This suggests that systems with very small velocity offsets between their multiple components may be rare, so our assumption of $\Delta v \gtrsim \sigma_{v}$ appears to be safe, even in the case of rotationally supported DSFGs.

Our fiducial simulations used a fixed number of 10 source clumps, randomly distributed across a galaxy of size $1 \mathrm{kpc}$. Our results do not appear to be as sensitive to these parameters as the clump size or linewidth, although this may depend on the details of the image morphology. For example, increasing the galaxy size allows the clumps to cover a larger portion of the caustic, which tightens the constraints on the macro model. However, it also increases the spacing between clumps, thereby degrading the sensitivity to low-mass substructure. However, Hezaveh et al. (2012b) showed that lensed DSFGs selected by a flux-cut in large area surveys are dominated by compact sources. The effect of changing the number of clumps is also somewhat unclear. In principle, a larger number of clumps should provide a larger number of constraints. However, since we hold fixed the total flux, increasing the clump number makes each clump fainter and lowers their signal-to-noise ratio. We found that for the fold configuration described above, decreasing the number of clumps from 10 to 5 somewhat improves the detection sensitivity, mainly by making each clump brighter. It is unclear whether this will hold for the other configurations as well. In the absence of a systematic study of all possible image configurations, we cannot say with any certainty how our substructure detection sensitivity will depend on the number and spread of the source clumps.

Although in this work we have focused on high excitation molecular lines emitted by the compact cores of star-forming clumps, the diffuse emission in low-excitation lines may also benefit from spatially resolved spectroscopy. Rotationally supported cold disks exhibit strong velocity gradients on the sky, meaning that the emission in narrow channels will originate from regions significantly smaller than the galaxy as a whole. In such cases, the observed velocity gradients in the lensed images of fast rotating cold gas reservoirs may show dips and peaks consistent with substructure lensing, complementing the high- $J$ line observations.

Our simulations have assumed that the mass distribution of the main lens may be described by the simple SIE profile, along with external shear. In principle, a broader set of mass models, allowing for different radial dependence or different angular dependence, could mask or mimic the effects of lowmass substructure. This possibility was explored by Kochanek \& Dalal (2004), and based on their results, we are confident that allowing for such deviations will have minimal impact on our derived substructure constraints. First, because the lensed images all occur at a similar radius from the lens center (i.e., the Einstein radius), varying the slope of the radial profile has little effect on the observables. The angular profile, in contrast, can produce significant effects on the lensed images. For quasar lenses, Kochanek \& Dalal found that allowing for low- $l$ angular multipoles (like $\cos (4 \theta)$ ) could degrade substructure detections somewhat. However, in cases where the lensed source produced an Einstein ring or extended arcs, the coefficients of such low- $l$ multipoles are so strongly constrained that they do not affect the detection significance of substructure. All the DSFG lenses that we have considered will have extended arcs, because the source objects are not pointlike, but instead are intrinsically extended, which means that low- $l$ multipoles will be strongly constrained by the data. Much higher- $l$ multipoles like $\cos (20 \theta)$ are actually substructure, merely with a different choice of basis. We are therefore confident that our simulations using SIE+shear for the macro model are representative of the substructure constraints that may be derived using ALMA data.

The calculations presented here were based on simulations of one hour observations using ALMA Cycle 1. Longer observing times would improve our quoted signal/noise ratios by $t^{1 / 2}$. In addition, the simulations presented here were specifically carried out for early science capabilities of ALMA with an array of only 32 antennas and a maximum baseline of $\sim 1 \mathrm{~km}$. The full ALMA array will consist of 64 antennas, which will increase the sensitivity by a factor of four. In addition, the longest baselines will extend as long as $\sim 16 \mathrm{~km}$, resulting in an angular resolution of $\sim 10$ milliarcsec at $850 \mu \mathrm{m}$. We can state with confidence that the full ALMA array will provide far greater substructure sensitivity than our Cycle 1 calculations have already found.

In this work, we have focused on ALMA observations of DSFGs, but, in principle, the methods used here are not limited to interferometric observations. For example, integral field spectroscopy (e.g., Alaghband-Zadeh et al. 2012; Barnabè et al. 2011; Förster Schreiber et al. 2011) of other lensed galaxies such Lyman break galaxies may benefit in the same way.

\section{CONCLUSION}

We have shown that it is possible to detect galactic dark matter substructure in the lens galaxies of lensed DSFG systems, using the early science capabilities of ALMA in Cycle 1. We found that the analysis of spatially resolved spectroscopic measurements of lensed sources can significantly improve substructure detection limits. In particular, we simulated ALMA Cycle 1 observations of molecular lines in these systems and calculated the detection significance for subhalos of different masses for various lensing configurations. We predict that with current ALMA capabilities and conservative assumptions about the morphology of DSFGs, there is a high probability of detecting at least one subhalo with $M \gtrsim 10^{8} M_{\odot}$ in every lensed $D S F G$. Only marginally more optimistic source morphologies allow us to go below this limit and explore $M \sim 10^{7} M_{\odot}$ dark matter subhalos. The full ALMA array, with $4 \times$ greater sensitivity and $16 \times$ higher angular resolution than what we have assumed, will assuredly improve our sensitivity to substructure beyond the calculations presented here. Given samples of $\sim 100$ lensed 
DSFGs discovered in large area millimeter surveys and the significantly enhanced capabilities of the completed ALMA, we are poised to revolutionize our understanding of low-mass dark matter substructure in coming years.

This work was supported by NASA under grant NNX12AD02G, by NSERC, the CRC program, and CIfAR. Y.D.H. is grateful for useful discussions with C. Fassnacht which considerably improved the $u v$-plane modeling. Y.D.H. acknowledges the support of FQRNT through International Training Program and Doctoral Research scholarships. N.D. gratefully acknowledges support by a Sloan Research Fellowship from the Alfred P. Sloan Foundation. We thank the SPT team for numerous useful discussions.

\section{REFERENCES}

Abazajian, K. 2006, PhRvD, 73, 063513

Alaghband-Zadeh, S., Chapman, S. C., Swinbank, A. M., et al. 2012, MNRAS, 424, 2232

Barnabè, M., Czoske, O., Koopmans, L. V. E., Treu, T., \& Bolton, A. S. 2011, MNRAS, 415, 2215

Blain, A. W. 1996, MNRAS, 283, 1340

Blain, A. W., Smail, I., Ivison, R. J., Kneib, J.-P., \& Frayer, D. T. 2002, PhR, 369,111

Bode, P., Ostriker, J. P., \& Turok, N. 2001, ApJ, 556, 93

Bothwell, M. S., Smail, I., Chapman, S. C., et al. 2012, MNRAS, 429, 3047

Browne, I. W. A., Wilkinson, P. N., Jackson, N. J. F., et al. 2003, MNRAS, 341,13

Carilli, C. L., Walter, F., Riechers, D., et al. 2011, arXiv:1105.1128

Carlberg, R. G., Richer, H. B., McConnachie, A. W., et al. 2011, ApJ, 731, 124

Carlstrom, J. E., Ade, P. A. R., Aird, K. A., et al. 2011, PASP, 123, 568

Cembranos, J. A. R., Feng, J. L., Rajaraman, A., \& Takayama, F. 2005, PhRvL, 95, 181301

Chapman, S. C., Smail, I., Windhorst, R., Muxlow, T., \& Ivison, R. J. 2004, ApJ, 611,732

Chen, J., Koushiappas, S. M., \& Zentner, A. R. 2011, ApJ, 741, 117

Chen, J., Kravtsov, A. V., \& Keeton, C. R. 2003, ApJ, 592, 24

Chen, J., Rozo, E., Dalal, N., \& Taylor, J. E. 2007, ApJ, 659, 52

Chiba, M., Minezaki, T., Kashikawa, N., Kataza, H., \& Inoue, K. T. 2005, ApJ, 627,53

Combes, F., Rex, M., Rawle, T. D., et al. 2012, A\&A, 538, L4

Cyr-Racine, F.-Y., \& Sigurdson, K. 2012, arXiv:1209.5752

Dalal, N., \& Kochanek, C. S. 2002, ApJ, 572, 25

Diemand, J., Kuhlen, M., \& Madau, P. 2007, ApJ, 667, 859

Diemand, J., Kuhlen, M., Madau, P., et al. 2008, Natur, 454, 735

Dodelson, S. 2003, Modern Cosmology (New York: Academic)

Downes, D., \& Solomon, P. M. 1998, ApJ, 507, 615

Engel, H., Tacconi, L. J., Davies, R. I., et al. 2010, ApJ, 724, 233

Fadely, R., \& Keeton, C. R. 2011, AJ, 141, 101

Förster Schreiber, N. M., Shapley, A. E., Genzel, R., et al. 2011, ApJ, 739, 45

Gao, Y., \& Solomon, P. M. 2004, ApJS, 152, 63

Greve, T. R., Bertoldi, F., Smail, I., et al. 2005, MNRAS, 359, 1165

Hezaveh, Y., Marrone, D., Faassnacht, C., et al. 2012a, ApJ, submitted

Hezaveh, Y. D., \& Holder, G. P. 2011, ApJ, 734, 52

Hezaveh, Y. D., Marrone, D. P., \& Holder, G. P. 2012b, ApJ, 761, 20

Hodge, J. A., Carilli, C. L., Walter, F., et al. 2012, ApJ, 760, 11
Hopkins, P. F., Cox, T. J., Hernquist, L., et al. 2012, MNRAS (arXiv:1206.0011) Kamionkowski, M., \& Liddle, A. R. 2000, PhRvL, 84, 4525

Kaplinghat, M. 2005, PhRvD, 72, 063510

Keeton, C. R., Burles, S., Schechter, P. L., \& Wambsganss, J. 2006, ApJ, 639, 1

Keeton, C. R., Gaudi, B. S., \& Petters, A. O. 2003, ApJ, 598, 138

Keeton, C. R., Gaudi, B. S., \& Petters, A. O. 2005, ApJ, 635, 35

Kochanek, C. S., \& Dalal, N. 2004, ApJ, 610, 69

Komatsu, E., Smith, K. M., Dunkley, J., et al. 2011, ApJS, 192, 18

Koopmans, L. V. E., Garrett, M. A., Blandford, R. D., et al. 2002, MNRAS, 334, 39

Kormann, R., Schneider, P., \& Bartelmann, M. 1994, A\&A, 284, 285

Kravtsov, A. 2010, AdAst, 2010, 8

Lagache, G., Puget, J.-L., \& Dole, H. 2005, ARA\&A, 43, 727

Lestrade, J.-F., Combes, F., Salomé, P., et al. 2010, A\&A, 522, L4

Loeb, A., \& Zaldarriaga, M. 2005, PhRvD, 71, 103520

MacLeod, C. L., Kochanek, C. S., \& Agol, E. 2009, ApJ, 699, 1578

Mao, S., \& Schneider, P. 1998, MNRAS, 295, 587

Metcalf, R. B. 2002, ApJ, 580, 696

Metcalf, R. B., \& Madau, P. 2001, ApJ, 563, 9

Metcalf, R. B., Moustakas, L. A., Bunker, A. J., \& Parry, I. R. 2004, ApJ, 607,43

Minezaki, T., Chiba, M., Kashikawa, N., Inoue, K. T., \& Kataza, H. 2009, ApJ, 697,610

Miranda, M., \& Macciò, A. V. 2007, MNRAS, 382, 1225

More, A., McKean, J. P., More, S., et al. 2009, MNRAS, 394, 174

Morgan, C. W., Hainline, L. J., Chen, B., et al. 2012, ApJ, 756, 52

Moustakas, L. A., \& Metcalf, R. B. 2003, MNRAS, 339, 607

Muñoz, J. A., Kochanek, C. S., \& Keeton, C. R. 2001, ApJ, 558, 657

Navarro, J. F., Ludlow, A., Springel, V., et al. 2010, MNRAS, 402, 21

Negrello, M., Hopwood, R., De Zotti, G., et al. 2010, Sci, 330, 800

Negrello, M., Perrotta, F., González-Nuevo, J., et al. 2007, MNRAS, 377, 1557

Petry, D., for the CASA Development Team 2012, in ASP Conference Series, ADASS XXI, ed. P. Ballester \& D. Egret (San Francisco, CA: ASP), 849

Rangwala, N., Maloney, P. R., Glenn, J., et al. 2011, ApJ, 743, 94

Reid, B. A., Samushia, L., White, M., et al. 2012, MNRAS, 426, 2719

Riechers, D. A., Cooray, A., Omont, A., et al. 2011, ApJ, 733, L12

Riechers, D. A., Weiß, A., Walter, F., \& Wagg, J. 2010, ApJ, 725, 1032

Rozo, E., Chen, J., \& Zentner, A. R. 2007, arXiv:0710.1683

Sanchez, A. G., Scoccola, C. G., Ross, A. J., et al. 2012, MNRAS, 425, 415

Schechter, P. L., \& Wambsganss, J. 2002, ApJ, 580, 685

Schneider, P., Ehlers, J., \& Falco, E. E. 1992, Gravitational Lenses (Berlin: Springer)

Seljak, U., Makarov, A., McDonald, P., \& Trac, H. 2006, PhRvL, 97, 191303

Sluse, D., Hutsemékers, D., Courbin, F., Meylan, G., \& Wambsganss, J. 2012, A\&A, 544, A62

Solomon, P. M., \& Vanden Bout, P. A. 2005, ARA\&A, 43, 677

Stacey, G. J., Geis, N., Genzel, R., et al. 1991, ApJ, 373, 423

Stadel, J., Potter, D., Moore, B., et al. 2009, MNRAS, 398, L21

Suzuki, N., Rubin, D., Lidman, C., et al. 2012, ApJ, 746, 85

Swinbank, A. M., Papadopoulos, P. P., Cox, P., et al. 2011, ApJ, 742, 11

Swinbank, A. M., Smail, I., Chapman, S. C., et al. 2010, MNRAS, 405, 234

Tacconi, L. J., Neri, R., Chapman, S. C., et al. 2006, ApJ, 640, 228

Vegetti, S., \& Koopmans, L. V. E. 2009, MNRAS, 392, 945

Vegetti, S., Lagattuta, D. J., McKean, J. P., et al. 2012, Natur, 481, 341

Vieira, J., Marrone, D., Chapman, S., et al. 2012, Natur, submitted

Vieira, J. D., Crawford, T. M., Switzer, E. R., et al. 2010, ApJ, 719, 763

Weiß, A., Downes, D., Neri, R., et al. 2007, A\&A, 467, 955

Xu, D. D., Mao, S., Wang, J., et al. 2009, MNRAS, 398, 1235

Yoon, J. H., Johnston, K. V., \& Hogg, D. W. 2011, ApJ, 731, 58

Younger, J. D., Fazio, G. G., Wilner, D. J., et al. 2008, ApJ, 688, 59

Zentner, A. R., \& Bullock, J. S. 2003, ApJ, 598, 49 